ISSN 1991- 8690

| الترقيم الدولي ·

website : http:// jsci.utq.edu.iq

Email: utjsci@utq.edu.iq

\title{
تصميم هوائي شريطي دائري محمل بحلقة معدنية
}

وائل عبد اللطيف*

زكي عبد الله احمد**

* رائد مسلم شعبان

$$
\begin{aligned}
& \text { *جامعة البصرة- مركز أبحاث البوليمر - قسم علوم المواد } \\
& \text { *** جامعة البصرة - كلية العلوم- قسم الفيزياء }
\end{aligned}
$$

(المدخص

تضمن البحث دراسـة نظريـة لتصميم هوائي شريطي دائري محمل بحلقة معدنية (CLAR) وحساب الهياكل الإثعاعية من خلال حل المعادلات التكاملية للمجال الكهربائي (EFIE) والمجال المغناطيسي (MFIE) حيث نم تحليل هذه المعادلات باستخدام طريقة العزوم (MoM) على اعتبار الهوائي

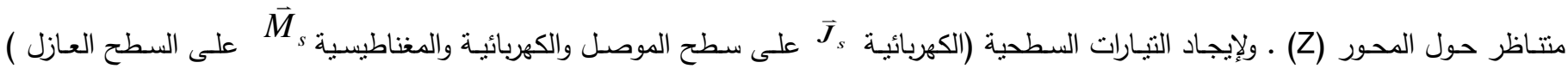
المسؤولة عن توليد المجالات الإثعاعية في الفضاء وللمركبتين ( متسلسلة فورير لامتلاك الجسم صفة التماثل ألتدويري ومن ثم اختيار مجموعة من الدوال الوزنية لإيجاد النظام الخطي عن طريق استخدام طريقة كالركن التي تشترط أن تكون الدوال الوزنية مساوية للمرافق العقدي للتيار (

\section{Design of a Circular Disc Microstrip Antenna Loaded By Annular Ring}

\author{
Raed M. AL-Saymaree* \\ Zeki A. Ahmed** \\ Wa'il A. Godaymi** \\ *Department of Material Science-Polymer Research Center-University of Basarh. \\ ** Department Physics- College of Science-University of Basarh.
}

\section{$\underline{\text { Abstract }}$}

The research contained in this thesis is a theoretical study of antenna design and calculation of the radiation fields using electric fields integral equation (EFIE) and magnetic field integral equation (MFIE). These equation have been converted, using the method of moments formulation of electromagnetic radiation in this these based on the bodies of revolution (BoR), which are generated by revolution a planar curve about an axis called axis of symmetry. To find an unknown electric current density on the surface of the conductor and both unknowns electric and magnetic density current on the surface of the dielectric which are responsible for the generation of radiation fields in the space for the components $(\mathrm{E} \theta, \mathrm{E} \varphi)$, the surface currents was represented by a set of basis functions that give the Fourier series because the body has a circular symmetry property and then select a set of weighted functions to find a linear system by using Galerkin method which requires that the weighted functions are equal to the complex conjugate of the current $\left(W=J^{*}\right)$. 


\section{النظرية}

استندت الدارسة النظرية لتحليل الإثتعاع الصادر من الهوائي

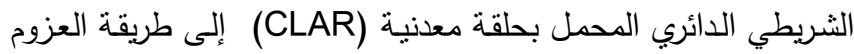

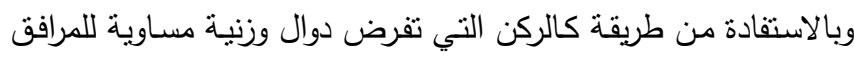

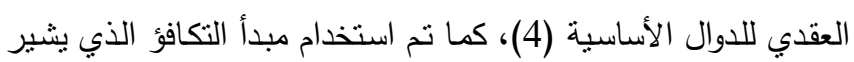

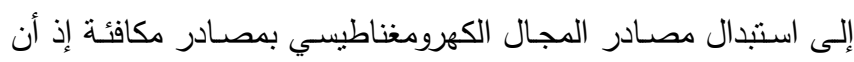
المصادر الكهرومغناطيسية المحتواة ضمن الدجم V والدحاط بالسطح

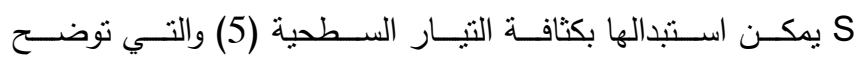

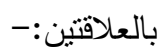

$\overline{\boldsymbol{J}}_{S}=\hat{n} \times \overline{\boldsymbol{H}}_{S}$

$\bar{M}_{S}=-\hat{n} \times \bar{E}_{S}$

بتطييق مبدأ النكافؤ على الهوائي الثـريطي الدائري المحمل بحلقة

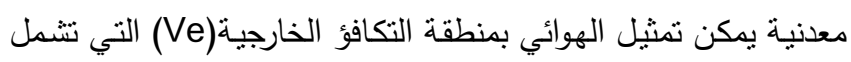
السطحين Sce(السطح الفاصل بين الموصل والفضاء الخارجي ) و

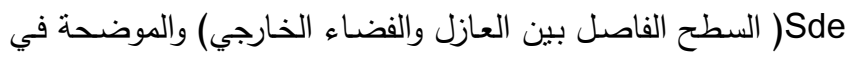
الثكل (2)، ومنطقة التكافؤ الداخلية (Vd) التي تتكون من السطحين

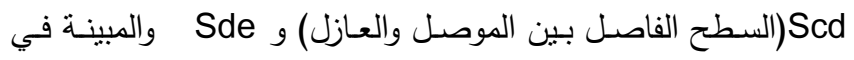

الثنكل (3).

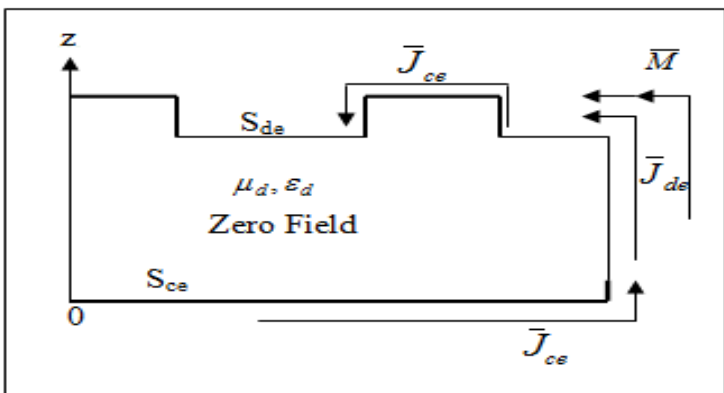

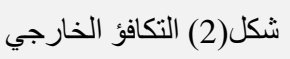

الهوائيات بصورة عامة ذات أهية كبيرة في مجال الاتصالات

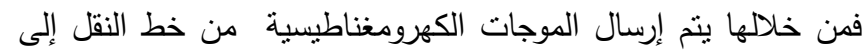
الفضاء الخارجي أو استلام تلك الموجات من الفضاء إلى خط النقات النقل، ويعد الهوائي الثريطي (MSA) من أكثر أنواع الهوائيات انتثاراً

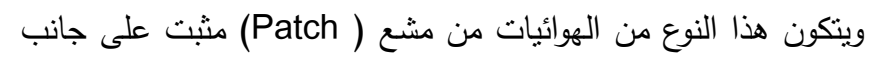
من شريحة عازلة كهربائيا (Dielectric Substrate) ويثبت على النى

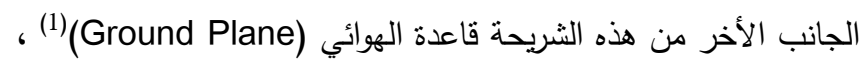

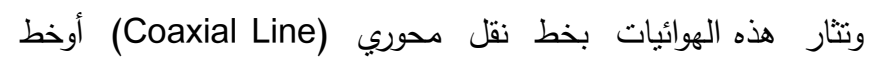
نقل شريطي (Microstrip Line) عندما يتم تصميم الهوائيات

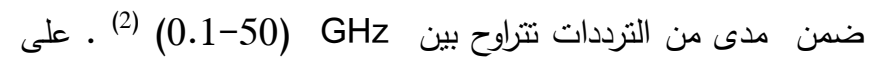
الرغم من الاستخدام الواسع للهوائيات الثريطية إلا أنها تعاني من نقاط

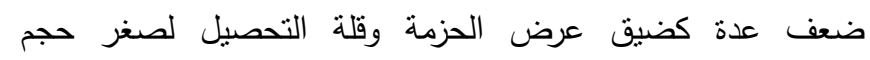
الهوائي(3) ولتحسين معاملات الهوائي سوف نعمل على تغير شكل التهل المشع للهوائي الثريطي الدائري من خلال إضافة حلقة معدنية موصلة

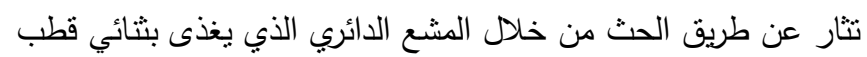

كهربائي كما مبين بالثكل (1) - (1).

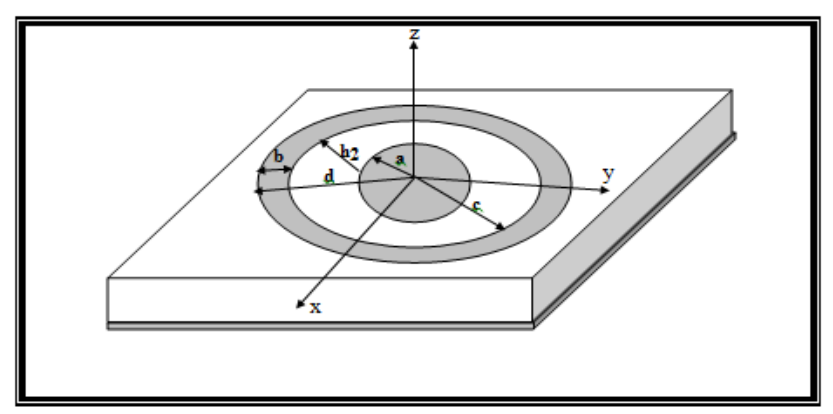

الشكل (1) هو ائي شريطي حلقي محمل بحلقة معدنية

حيث إن (d) يمثل نصف القطر الخارجي للحلقة بينما (c) يمثل نصف

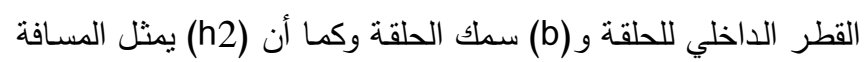
الفاصلة بين الحلقة والقرص و (a) تمثل نصف قطر القرص. 
حيث والمغناطيسي على النقالي المتولاين نتيجة التيار الكهربائي المحتث والثنار المغناطيسي المحتث الموصوفة بالمعاملات ( النكافؤ $e$

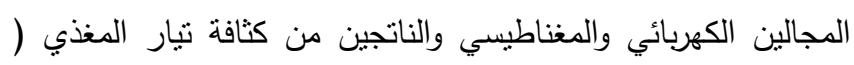

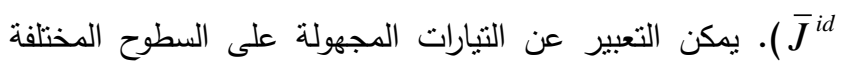

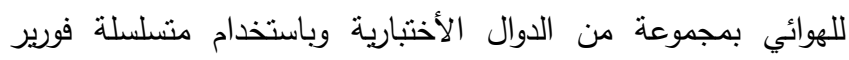
والدوال التنلثية (8) لنقريب كثافة النثار على السطح S S بالصيغة

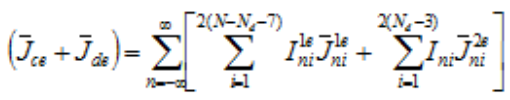

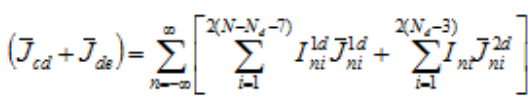

$$
\begin{aligned}
& \bar{M}=\eta_{e} \sum_{n=-\infty}^{\infty} \sum_{i=1}^{2\left(N_{k}-3\right)} K_{k=1} \bar{M}_{k i}
\end{aligned}
$$$$
\text { وبتعويض المعادلات (6) في المعادلات (5) نحصل على :- }
$$

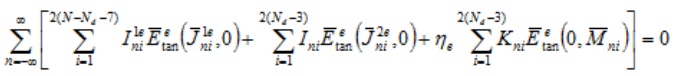$$
\text { on } \mathrm{S}_{\mathrm{ce}} \quad \ldots . .(7-\mathrm{a})
$$

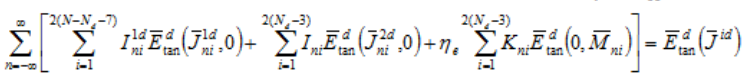$$
\text { , on } \mathrm{S}_{\mathrm{cd}}
$$

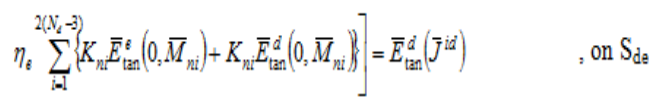

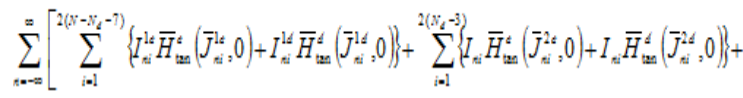

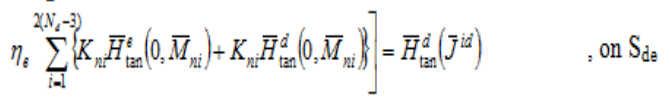

وفي دراستتا الحالية استخدنا طريقة كالركن وفي هذه الطريقة تكون

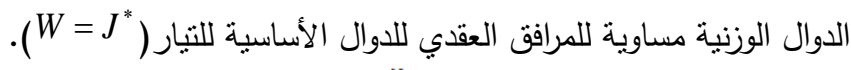
$\bar{W}(\bar{r})=\bar{W}^{t}(t, \phi)+\bar{W}^{\phi}(t, \phi)=\sum_{m=-\infty}^{\infty} \sum_{i=1}^{N-10}\left[\bar{W}_{m i}^{t}(t, \phi)+\bar{W}_{m i}^{\phi}(t, \phi)\right]$

$$
\bar{W}_{m i}^{t}(t, \phi)=\hat{u}_{t} f_{i}(t) e^{-j m \phi}
$$

$\bar{W}_{m i}^{\phi}(t, \phi)=\hat{u}_{\phi} f_{i}(t) e^{-j m \phi}$

وباستخدام الضرب العددي للدوال الوزنية مـع المعادلات (7) نحصل

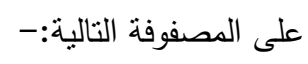

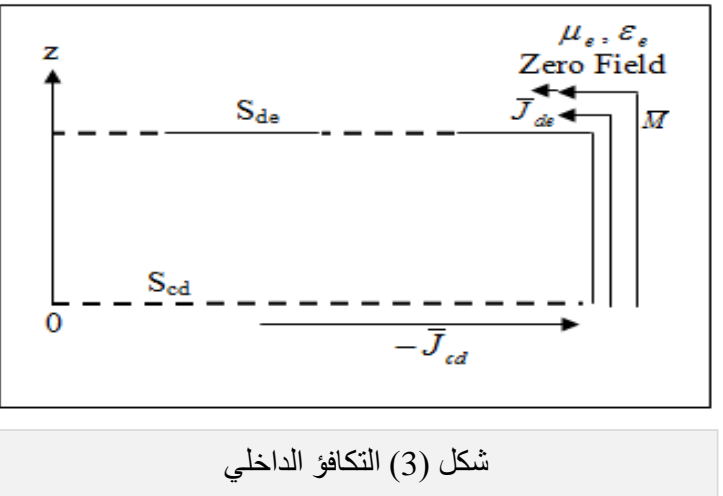

أن الهوائي الثـريطي يتكـون مـن مـواد موصـلة وعازلـة وبالتـالي فأنها تحتوي على نوعين من شروط المسألة على كل من العازل والموصل موصل (6)، وهذه الثروط تفرض تلاشي مركبات المجال الكهربائي المماسية

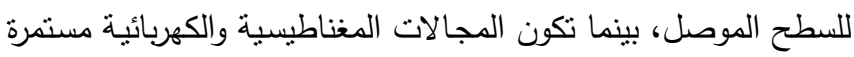
عند سطح العازل(7) ويمكن وصفها كالتالي :$\hat{n} \times \bar{E}^{e}=0 \quad$ on $\mathrm{S}_{\mathrm{ce}} \ldots . .(3-\mathrm{a})$ $\hat{n} \times \bar{E}^{d}=0 \quad$ on $\mathrm{S}_{\mathrm{cd}} \ldots \ldots(3-\mathrm{b})$ $\hat{n} \times \bar{E}^{d}=\hat{n} \times \bar{E}^{e} \quad$ on $\mathrm{S}_{\mathrm{de}} \ldots \ldots(3-\mathrm{c})$ $\hat{n} \times \bar{H}^{d}=\hat{n} \times \bar{H}^{e} \quad$ on $\mathrm{S}_{\mathrm{de}} \ldots . .(3-\mathrm{d})$ وحسب مبدأ التكافؤ الخارجي والداخلي نجد كثافة التبارات كما يلي :$\begin{array}{ll}\bar{J}_{c e}=\hat{n} \times \bar{H}^{e} & \text { on } \mathrm{S}_{\mathrm{ce}} \ldots . .(4-\mathrm{a}) \\ \bar{J}_{c d}=\hat{n} \times \bar{H}^{d} & \text { on } \mathrm{S}_{\mathrm{cd}} \ldots . .(4-\mathrm{b}) \\ \bar{J}_{d e}=\hat{n} \times \bar{H}^{e} & \text { on } \mathrm{S}_{\mathrm{de}} \ldots . .(4-\mathrm{c}) \\ \bar{M}=-\hat{n} \times \bar{E}^{e} & \text { on } \mathrm{S}_{\mathrm{de}} \ldots . .(4-\mathrm{d})\end{array}$ إن التيار الكهربائي J لتولد على السطحين الموصل والعازل في حين يتولد التيار المغناطيسي على السطح العازل فقط ، وعليه فعند تطبيق مبدأ التكافؤ على منطقتي التكافؤ الخارجية والداخلية للمسألة

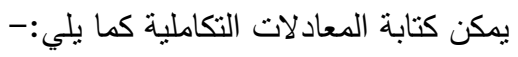

$\left.\begin{array}{l}\hat{n} \times \bar{E}^{e}\left(\bar{J}_{c \varepsilon}+\bar{J}_{d e}, \bar{M}\right)=0 \\ \hat{n} \times H^{c}\left(J_{c e}+J_{d s}, M\right)=0\end{array}\right]$ on $\mathrm{S}_{\mathrm{ce}}$ and $\mathrm{S}_{\mathrm{de}}$

$\left.\begin{array}{l}\hat{n} \times \bar{E}^{d}\left(-\bar{J}_{t d}-\bar{J}_{d t},-\bar{M}\right)+\hat{n} \times \bar{E}^{d}\left(\bar{J}^{i d}, 0\right)=0 \\ \hat{n} \times \bar{H}^{d}\left(-\bar{J}_{t d}-\bar{J}_{d t},-\bar{M}\right)+\hat{n} \times \bar{H}^{d}\left(\bar{J}^{i d}, 0\right)=0\end{array}\right] \mathrm{S}_{\mathrm{cd}}$ and $\mathrm{S}_{\mathrm{de}}$ 
للهوائي :- وبعد التأكد من صحة البرامج المستخدم لبرمجة التحليل الرياضي للهوائي قيد الدراسة ولغرض تحسين معاملات الهوائي قمنا

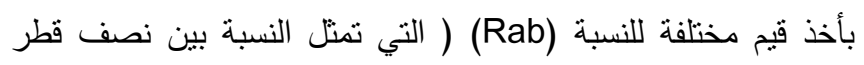

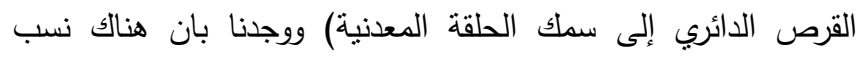

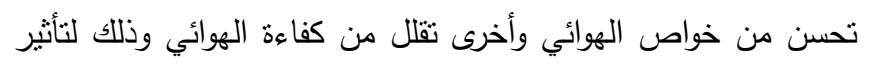

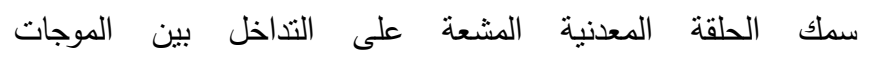

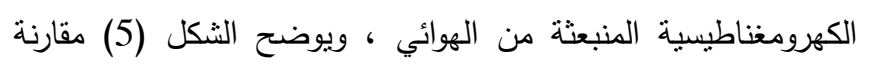
الهيكل الإشعاعي لهوائي شريطي دائري محمل بحلقة مشعة (CLAR) للمستوي الأساسي (E-PLANE) عندما ينتشر بداخلة النمط 11 النمي

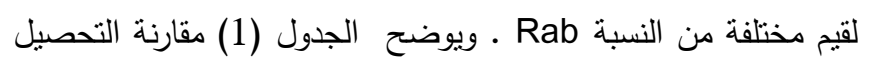

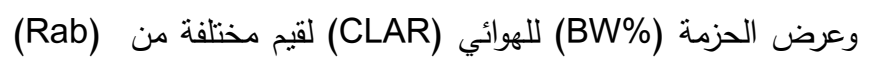

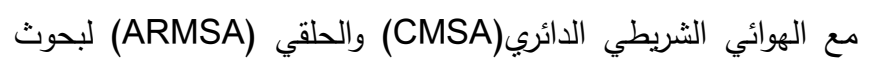

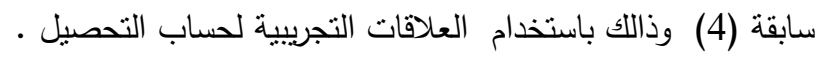
$G(\mathrm{~dB})=10 \log \left(\frac{36800}{H P_{H^{\circ}} H P_{F^{\circ}}}\right)$

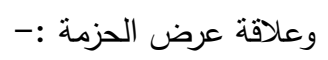

$\mathrm{BW}_{\text {Narrowband }} \%=\left(\frac{F_{u}-F_{l}}{F_{r}}\right) * 100 \%$

حيث نلاحظ أن قيمة عرض الحزمة تكون الأفضل للنسبة (Rab=10)

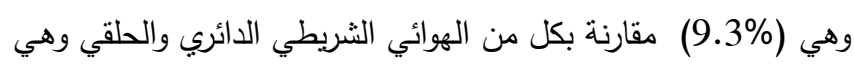
(1.1\%) و (0.7\%) على التوالي، إما بالنسبة للتحصيل فأن أفضل التصل النسب كانت (Rab=2) وهـي (R.28dB) مقارنـة بكل مـن الهوائي الثريطي الدائري والحلقي وهي (6.8\%) و (6.1\%) على (6.1\%) على التوالي.

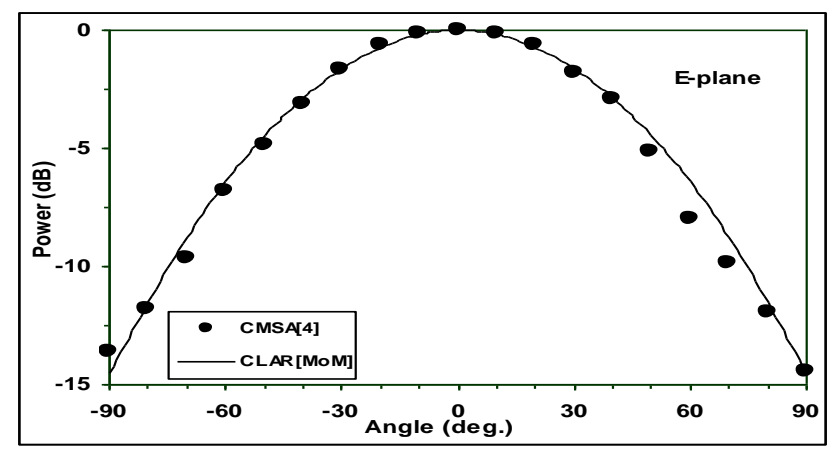

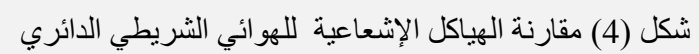
المحمل بحلقة معدنية و الهو ائي الثريطي الدائري عندما ينتشئر بداخلة الثنة النمط

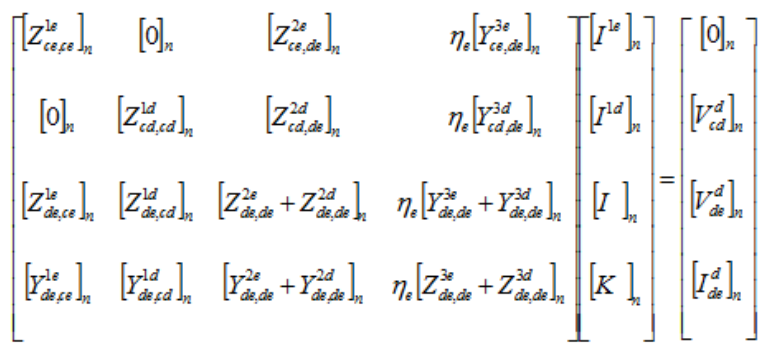

$$
\text { و التي يمكن اختصار ها بالثكل التالي :- }
$$

$\left[\bar{T}_{n m}\right]\left[\bar{I}_{n}\right]=\left[\bar{V}_{n}\right]$

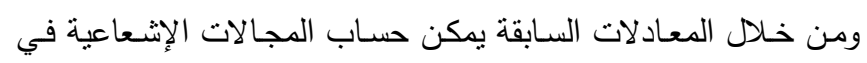

منطقة الإشعاع (المجال البعيد) من خلال المعادلات التالية (9) :-

$$
\begin{aligned}
& E_{\theta}=-\frac{j w \mu_{\varepsilon}}{4 \pi r_{o}} e^{-j E_{e} r_{0}} F_{1}\left(\theta_{\theta}, \phi_{0}\right) \\
& E_{\phi}=-\frac{j w \mu_{\varepsilon}}{4 \pi r_{o}} e^{-j K_{\tau_{0}}} F_{2}\left(\theta_{o}, \phi_{0}\right)
\end{aligned}
$$

حيـث أن والمعرفة كما يلي :-

$F_{1}\left(\theta_{0}, \phi_{0}\right)=\iint_{s}\left(\bar{J}\left(\bar{r}^{\prime}\right) \cdot \hat{\theta}+\frac{1}{\eta_{\varepsilon}} \bar{M}\left(\bar{r}^{\prime}\right) \cdot \hat{\phi}\right) e^{-j K_{\theta_{e}} \cdot \sigma^{\prime \prime}} d s$

$F_{2}\left(\theta_{o}, \phi_{0}\right)=\int_{s}\left(\bar{J}\left(\bar{r}^{\prime}\right) \cdot \hat{\phi}-\frac{1}{\eta_{\varepsilon}} \bar{M}\left(\bar{r}^{\prime}\right) \cdot \hat{\theta}\right) e^{-j K_{\ell_{e}} \cdot r} d s$

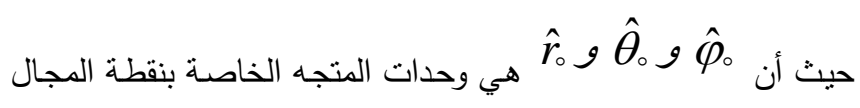
البعيد.

\section{الانتائج والمناقشية}

يوضح الثكل (4) مقارنة الهيكل الإثعاعي لهوائي شريطي

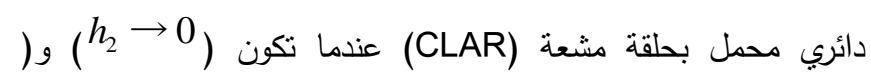
) مع هوائي شريطي دائري (CMSA) وذلك للتأكد من صحة (CMA 0 البرامج المستخدمة لوصف المعادلات التي حصلنا عليها من خلال

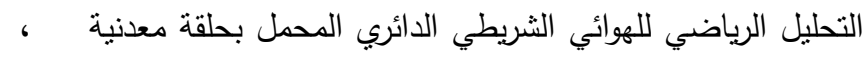

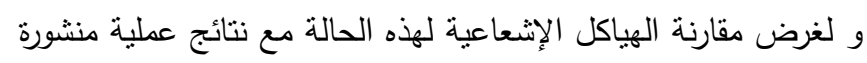

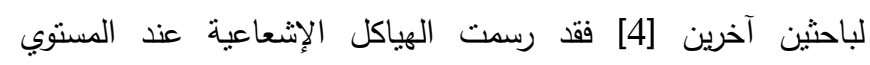
الأساسي الكهربائي E-plane للنمط النتائج التي توصلنا إليها والنتائج العملية المنشورة وذلك لان لان طريقة

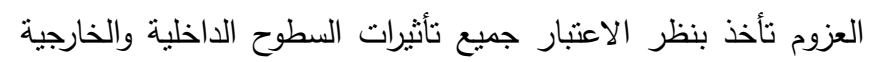


اللهوائي قيد الدراسة كان عند النسبة ( $\left(\mathrm{d}_{\mathrm{ab}}=2\right.$ وهي (8.28dB) ، إما عرض الحزمة فكانت النسبة (Rab $\left(R_{\text { }}\right.$ هي الأفضل وهي (9.3\%).

\section{References}

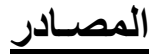

1. Bahl, I . J . and Bahartia, P., "Microstrip antennas", Artech House, Inc., 1980.

2. Deschamps, G.A., "Microstrip Microwave antennas",presented at the 3rd USAF Symposium on Antennas, 1953.

3. Howell,J.Q., "Microstrip antennas", IEEE AP-S Int. Symp.Digest, 1972,pp.177-180.

4. James, J. R. and Hall, P. S., "Handbook of microstrip antennas", Peter Peregrines, London, U. K., 1989.

5. Harrington, R . F , " Field computation by moment methods ", Macmillan, New York, 1993.

6. Timothy, E . D, and Christos G. C," Integral equation analysis of dielectric and conducting bodies of revolution in the presence of arbitrary surfaces" IEEE Transaction on Antennas and propagation, Vol. 43. No. 7, pp.674-680,1995.

7. Resende, U. C., Moreira, F. J. S.," Efficient evaluation of singular integrals in moment methods applied to solve scattering problems from bodies of revolution", IEEE ,2006.

8. Jian B. , Daoxiang W. , and Kai N.Y.," Radiation and scattering from bi-isotropic bodies of revolution ", IEEE 2007.

9. Resende, U. C., Moreira, F. J. S.," Efficient evaluation of singular integrals in moment methods applied to solve scattering problems from bodies of revolution", IEEE ,2006.
$\left(\mathrm{R}_{\mathrm{g}}=0.31 \lambda, \mathrm{f}_{\mathrm{r}}=794 \mathrm{MH}_{\mathrm{Z}} \mathrm{R}_{\mathrm{g} 1}=0.01 \lambda, \mathrm{h}=0.0397 \lambda, \mathrm{a}_{1}=0.0077 \lambda\right.$, $\left.\mathrm{a}_{3}=0.0077, \varepsilon_{\mathrm{r}}=2.32 \varphi_{\mathrm{f}}=0^{\circ}, \rho_{\mathrm{f}}=0.05 \lambda, \mathrm{R}_{\mathrm{ab}}=55\right)$

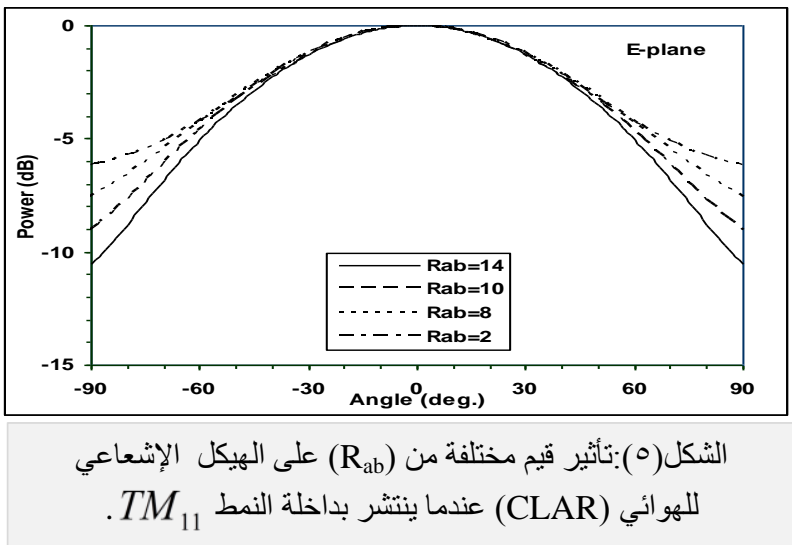

$\left(\mathrm{R}_{\mathrm{g}}=0.31 \lambda, \mathrm{f}_{\mathrm{r}}=374 \mathrm{MH}_{\mathrm{Z}} \mathrm{R}_{\mathrm{g} 1}=0.01 \lambda, \mathrm{h}=0.0397 \lambda, \mathrm{a}_{1}=0.0077 \lambda\right.$, $\left.\mathrm{a}_{3}=0.0077, \varepsilon_{\mathrm{r}}=2.32 \varphi_{\mathrm{f}}=0^{\circ}, \rho_{\mathrm{f}}=0.05 \lambda\right)$

جدول(1) مقارنة الهو ائي(CLAR) مع (CLMA) الهو ائيين(CMSA)و(ARMSA) للتحصيل و عرض الحزمة لقيم مختلفة من (hA) (h)

\begin{tabular}{|l|c|c|c|c||}
\hline Antenna Type & $\begin{array}{c}\mathrm{HP}_{\mathrm{H}^{\circ}} \\
(\mathrm{deg})\end{array}$ & $\begin{array}{c}\mathrm{HP}_{\mathrm{E}^{\circ}} \\
(\mathrm{deg})\end{array}$ & $\begin{array}{c}\text { Directive } \\
\text { gain (dB) }\end{array}$ & $\begin{array}{c}\mathrm{BW} \% \\
\mathrm{~S}=1.25\end{array}$ \\
\hline CMSA[4] & 80 & 100 & 6.8 & 1.1 \\
\hline ARMSA[4] & 81 & 103 & 6.1 & 0.7 \\
\hline CLAR $\left(\mathrm{R}_{\mathrm{ab}}=14\right)$ & 90 & 92 & 6.4 & 1.69 \\
\hline CLAR $\left(\mathrm{R}_{\mathrm{ab}}=10\right)$ & 80 & 96 & 6.8 & 9.3 \\
\hline CLAR $\left(\mathrm{R}_{\mathrm{ab}}=8\right)$ & 68 & 98 & 7.4 & 6.1 \\
\hline CLAR $\left(\mathrm{R}_{\mathrm{ab}}=2\right)$ & 58 & 92 & 8.28 & 3.84 \\
\hline
\end{tabular}

\section{الاستنتاجات}

طريقة العزوم التي تعدّ من أفضل الطرائق العددية لتحليل الهوائي الثريطي الدائري المحمل بحلقة معدنية لإعطائها نتائج دقيقة وكان ذللك واضحا من خلال التقارب بين الهيكل الإشعاعي للهوائي قيد الدراسة مع

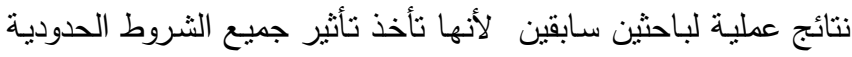
الخاصـة بجسـم الهـوائي ، ومـن نتـائج الهياكل الإثـعاعية للهـوائي لئي

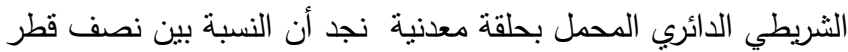

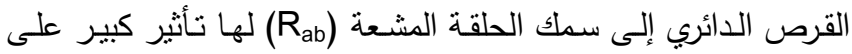

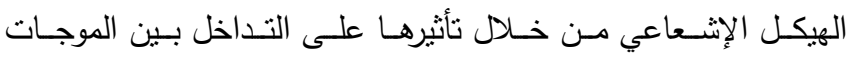
الكهرومغناطيسية المشعة والمستلمة ،حيث نلاحظ أن أفضل تحصيل 